\title{
HIV serostatus disclosure is not associated with safer sexual behavior among HIV-positive men who have sex with men (MSM) and their partners at risk for infection in Bangkok, Thailand
}

\author{
Nneka Edwards-Jackson', Nittaya Phanuphak ${ }^{2,3}$, Hong Van Tieu ${ }^{1,4}$, Nitiya Chomchey ${ }^{2}$, Nipat Teeratakulpisarn ${ }^{3}$, \\ Wassana Sathienthammawit ${ }^{3}$, Charnwit Pakam ${ }^{3}$, Nutthasun Pharachetsakul ${ }^{3}$, Magdalena E Sobieszczyk', \\ Praphan Phanuphak ${ }^{3,5}$ and Jintanat Ananworanich ${ }^{2,3,5^{*}}$
}

\begin{abstract}
Background: The relationship between HIV serostatus disclosure and sexual risk behavior is inconsistent across studies. As men who have sex with men (MSM) are emerging as the key affected population in Bangkok, Thailand with reported HIV prevalence of 30\%, we assessed whether HIV disclosure is associated with protected sex in this population.

Methods: A risk behavior questionnaire was administered using Audio Computer-Assisted Self-Interviewing (ACASI) to determine whether HIV serostatus disclosure was associated with protected sex in 200 HIV-positive MSM in Bangkok. HIV serostatus disclosure to the most recent sexual partner prior to or at the time of the sexual encounter was assessed. Protected sex was defined as insertive or receptive anal intercourse with a condom at the most recent sexual encounter.
\end{abstract}

Results: The mean age was 30.2 years, CD4 was 353 cells $/ \mathrm{mm}^{3}$, and one-third was on antiretroviral therapy. At the most recent sexual encounter, HIV serostatus disclosure rate was low (26\%); $60.5 \%$ of subjects had not discussed their serostatus at all, while 5.5\% had not revealed their true serostatus. Seventeen percent reported unprotected anal intercourse and about half had sex with their primary partners. The serostatus of the most recent sexual partner was HIV-positive in $19.2 \%$, HIV-negative in $26.4 \%$, and unknown in $54.4 \%$ of subjects. There was no association between disclosure and protected sex, with 41 of 48 (85.4\%) disclosers and 104 of 126 (82.5\%) of non-disclosers reported protected sex $(p=.65)$. Subjects with HIV-positive partners were less likely to report protected sex overall $(20$ of $33,60.6 \%$ ) compared to those with HIV negative ( 82 of $96,85.4 \%$ ) or unknown (41 of $45,91.1 \%)$ partners $(p=.001)$. Age (27-32 years vs. $\leq 26$ years, $p=.008)$, primary partner status $(p<.001)$, and HIV-positive serostatus of sexual partner $(p<.001)$ were significantly associated with disclosure in the multivariate analyses.

Conclusion: Rates of HIV disclosure to sexual partners by HIV-positive MSM in Bangkok are low. Despite low rates of HIV serostatus disclosure, most HIV-positive MSM reported protected sex with their partners at risk for infection. Future studies should focus on understanding barriers to disclosure and factors driving risk behavior amongst MSM in Thailand.

Keywords: HIV-positive, Serostatus disclosure, Men who have sex with men, Thailand

\footnotetext{
* Correspondence: Jintanat.a@searchthailand.org

${ }^{2}$ SEARCH, 104 Rajdumri Road, Pathumwan, Bangkok 10330, Thailand

${ }^{3}$ The Thai Red Cross AIDS Research Center, 104 Rajdumri Road, Pathumwan, Bangkok 10330, Thailand

Full list of author information is available at the end of the article
} 


\section{Background}

Men who have sex with men (MSM) have emerged as a population at high risk for HIV-1 infection in Thailand. In 2003, a community-based study among MSM in Bangkok demonstrated HIV prevalence as high as17.3\% [1]. Followup studies showed this prevalence had increased to $28.3 \%$ in 2005 and $30.8 \%$ in 2007 [1,2]. An examination of the factors that influence the transmission of HIV in the Thai MSM community is needed to help guide prevention efforts. Disclosure of HIV serostatus to sexual partners may serve as an important prevention intervention in this population.

HIV serostatus disclosure has been well studied in Western countries. Although various factors have been shown to influence the likelihood of disclosure among HIV-positive MSM [3-5], the relationship between HIV serostatus disclosure and sexual risk behavior, has been inconsistent across studies. Some studies have revealed that disclosers are more likely to practice safer sexual behaviors $[5,6]$ while others have shown no association $[3,7]$.

Data are lacking on HIV serostatus disclosure among HIV-positive MSM in Thailand. We therefore aimed to determine whether HIV disclosure was associated with protected sex, and to describe the factors associated with disclosure.

\section{Results}

Table 1 outlines the demographics and behavioral characteristics of the study population. Mean age was 30.2 years. Mean duration of known HIV infection was 3 years with a mean CD4 T cell count of 353 cells $/ \mathrm{mm}^{3}$. About onethird of men was on antiretroviral therapy (ART). Subjects reported a mean of 3.3 male sexual partners in the past 3 months. At their most recent sexual encounter, $17.2 \%$ and $70.9 \%$ of men reported engaging in unprotected anal and oral intercourse, respectively; $7.0 \%$ and $9.0 \%$ either did not respond or denied that sexual route at their last sexual encounter, respectively. Only $26.0 \%$ of participants had disclosed their HIV-positive serostatus to their partner prior to that encounter; $60.5 \%$ of subjects had not discussed their serostatus at all, while $5.5 \%$ had not revealed their true serostatus. About half (51.1\%) of the most recent sexual partners were primary partners. The serostatus of the most recent sexual partner was HIV-positive in $19.2 \%$, HIV-negative in $26.4 \%$, and unknown in $54.4 \%$ of subjects.

Reported rates of protected anal intercourse were relatively high, but there was no association between disclosure and protected sex; 41 of 48 (85.4\%) disclosers and 104 of $126(82.5 \%)$ of non-disclosers reported protected sex $(\mathrm{p}=0.65$; OR $1.23,95 \%$ CI $[0.49,3.12]$; adjusted OR 1.58, 95\% CI $[0.47,5.35]$ ) (Table 2). The most common behavioral pattern of disclosure and sexual behavior was non-disclosure/protected sex, which
Table 1 Demographic and sexual behavior data $(\mathrm{N}=200)^{\mathrm{a}}$

\begin{tabular}{|c|c|}
\hline \multicolumn{2}{|l|}{ Demographics } \\
\hline Age, years, $\mathrm{n}(\%)$ & \\
\hline$\leq 25$ & $57(28.5)$ \\
\hline $26-30$ & $64(32.0)$ \\
\hline $31-35$ & $37(18.5)$ \\
\hline$>35$ & $42(21.0)$ \\
\hline \multicolumn{2}{|l|}{ Education level, n (\%) } \\
\hline High school and below & $37(18.5)$ \\
\hline Vocational/technical training & $25(12.5)$ \\
\hline College or above & $134(67.0)$ \\
\hline \multicolumn{2}{|l|}{ Monthly income, $\mathrm{n}(\%)^{\mathrm{b}}$} \\
\hline$<10,000$ Thai baht & $61(30.5)$ \\
\hline 10,000-20,000 Thai baht & $81(40.5)$ \\
\hline$>20,000$ Thai baht & $48(24.0)$ \\
\hline \multicolumn{2}{|l|}{ Occupation, n (\%) } \\
\hline Student & $29(14.8)$ \\
\hline Employed & $142(72.4)$ \\
\hline Unemployed & $23(11.7)$ \\
\hline Retired & $2(1.0)$ \\
\hline \multicolumn{2}{|l|}{ Current household, $\mathrm{n}(\%)$} \\
\hline Living with spouse or primary partner & $30(15.0)$ \\
\hline Living with roommate(s) & $35(17.5)$ \\
\hline Living with relatives & $68(34.0)$ \\
\hline \multicolumn{2}{|l|}{ Years since HIV diagnosis, n (\%) } \\
\hline $0-1$ year & $93(50.5)$ \\
\hline $2-4$ years & $52(26.0)$ \\
\hline 5 or more years & $39(19.5)$ \\
\hline \multicolumn{2}{|l|}{ Current CD4 count, cells/mm³ $\mathrm{n}(\%)$} \\
\hline$\leq 200$ & $28(14.0)$ \\
\hline $201-350$ & $62(31.0)$ \\
\hline $351-500$ & $55(27.5)$ \\
\hline$>500$ & $30(15.0)$ \\
\hline Current anti-retroviral use, $\mathrm{n}(\%)$ & $66(33.0)$ \\
\hline History of AIDS-defining illness, $\mathrm{n}(\%)^{c}$ & $32(16.0)$ \\
\hline \multicolumn{2}{|c|}{ Sexual identity and behavior } \\
\hline \multicolumn{2}{|l|}{ Sexual orientation, n (\%) } \\
\hline Homosexual & $171(85.5)$ \\
\hline Bisexual & $16(8.0)$ \\
\hline Uncertain of sexuality & $4(3.0)$ \\
\hline \multicolumn{2}{|c|}{ Number of male sexual partners in the past 3 months, $n(\%)^{d}$} \\
\hline 1 & $103(51.5)$ \\
\hline $2-5$ & $73(36.5)$ \\
\hline $6-10$ & $10(5.0)$ \\
\hline$>10$ & $11(5.5)$ \\
\hline
\end{tabular}


Table 1 Demographic and sexual behavior data $(\mathrm{N}=200)^{\mathrm{a}}$ (Continued)

\begin{tabular}{ll}
\hline Type of most recent sexual partner $^{\mathrm{e}}$ & \\
Primary or main partner & $93(51.1)$ \\
Secondary partner & $89(48.9)$ \\
\hline HIV serostatus of most recent sexual partner & \\
Known HIV-positive & $35(19.2)$ \\
Known HIV-negative & $48(26.4)$ \\
Serostatus-unknown & $99(54.4)$
\end{tabular}

Disclosure of HIV status to most recent sexual partner, $\mathrm{n}(\%)^{\mathrm{e}}$

Disclosure of HIV-positive status

$52(26.0)$

No disclosure

$121(60.5)$

Disclosure of HIV-negative or unknown status 11 (5.5)

Disclosure by someone else

Partner awareness of subject's HIV-positive status ${ }^{\mathrm{e}}$

Partner aware of HIV-positive status

$56(28.0)$

Partner unaware

$125(62.5)$

Uncertain of partner's awareness

Unprotected intercourse, $\mathrm{n}(\%) \mathrm{g}$

Unprotected anal intercourse

Unprotected oral intercourse

$129(70.9)$

${ }^{a}$ All percentages presented were calculated using $\mathrm{N}=200$ to include nonresponders.

${ }^{\mathrm{b}}$ Average monthly household income in Thailand in 2007 was 18,660 baht, or approximately 570 USD (National Statistical Office, 2008).

'Centers for Disease Control and Prevention Classification.

${ }^{\mathrm{d}}$ No female sexual partners were reported.

ePrimary or main partners were partners whom one lives with or spends a lot of time with, and to whom one feels an emotional commitment. Secondary partners were all non-primary sexual partners.

fPartner awareness was assessed by the question, "Did your most recent sexual partner know you are infected with HIV before you had sex?". Disclosure was assessed with the follow-up question, "Did you discuss your HIV status with this person before you had sex?".

was reported by $60.0 \%$ of subjects. The proportions of subjects who reported disclosure/protected sex, disclosure/ unprotected sex, and non-disclosure/unprotected sex were $23.6 \%, 4.0 \%$, and $12.6 \%$, respectively.

Among subjects with HIV-positive partners $(\mathrm{n}=32)$, disclosers were more likely to report protected sex than nondisclosers; 17 of 22 (77.3\%) disclosers versus 2 out of 10 (20.0\%) non-disclosers reported protected sex $(\mathrm{p}=0.005$;
OR 13.6, 95\% CI [2.15, 85.9]; adjusted OR 13.0, 95\% CI $[1.28,125])$ (Table 2). There was no association between disclosure and protected sex among subjects with serodiscordant and serostatus-unknown partners as most of these men reported protected sex with partners regardless of whether or not they disclosed. Among subjects with HIV-negative partners, 10 of 10 (100\%) disclosers reported protected sex versus 30 of 31 (96.8\%) of nondisclosers $(\mathrm{p}=0.76$, odds ratios not calculated due to small number of subjects engaging in unprotected sex). Among subjects whose partner's HIV serostatus was unknown, 10 of 11 (90.0\%) disclosers reported protected sex versus 67 of $80(83.8 \%)$ of non-disclosers $(\mathrm{p}=0.54$; OR $1.94,95 \%$ CI $[0.23,16.5]$; adjusted OR $1.74,95 \%$ CI [0.20, 18.2]) (Table 2). Of note, subjects with HIV-positive partners were less likely to report protected sex overall (20 of $33,60.6 \%$ ) compared to those with HIV negative (82 of 96 , $85.4 \%)$ or unknown (41 of $45,91.1 \%)$ partners $(\mathrm{p}=.001$ ). There was no association between disclosure and protected sex among other subgroups of age or partner type.

Of the factors examined (age, partner type and HIV serostatus, number of sexual partners in the past 3 months, sexual orientation and level of outness [8], alcohol and drug use at last sexual encounter, years since HIV diagnosis, CD4 count, current antiretroviral use, history of AIDS diagnoses, and level of HIV knowledge [9]), only age (27-32 years vs. $\leq 26$ years, $\mathrm{p}=0.008$, adjusted OR 6.21 $95 \%$ CI $[2.0,19.6])$, primary partner status $(\mathrm{p}<0.001$, adjusted OR 16.6 95\% CI [5.0, 55.5]), and HIV-positive serostatus of sexual partner $(\mathrm{p}<0.001$, adjusted OR 13.6 95\% CI $[4.3,42.4])$ were significantly associated with disclosure in the multivariate analyses.

\section{Discussion}

In this study, we demonstrated low rates of HIV serostatus disclosure to sexual partners by HIV-positive MSM in Bangkok. Factors associated with HIV serostatus disclosure included older age, primary partner status, and HIV-positive serostatus of sexual partner. There was no significant association between disclosure of HIV serostatus and self-reported protected sex except in men who have HIV positive partners. Furthermore, despite a lack of disclosure, most HIV-positive men reported

Table 2 Relationship between HIV serostatus disclosure and protected sex ${ }^{a}$

\begin{tabular}{lccccc}
\hline & Disclosers N (\%) & Non-disclosers N (\%) & p & OR (95\% Cl) & Adjusted OR (95\% Cl) \\
\hline Overall $(\mathrm{N}=174)$ & $41(85.4)$ & $104(82.5)$ & 0.65 & $1.23(0.49,3.12)$ & $1.58(0.47,5.35)^{\mathrm{b}}$ \\
\hline Subjects with HIV-positive partners $(\mathrm{N}=32)$ & $22(77.3)$ & $2(20.0)$ & 0.005 & $13.6(2.15,85.9)$ & $13.0(1.28,125)^{\mathrm{c}}$ \\
\hline Subjects with HIV-negative partners $(\mathrm{N}=41)$ & $10(100)$ & $30(96.8)$ & 0.76 & - $^{\mathrm{d}}$ & $-{ }^{\mathrm{d}}$ \\
\hline Subjects with HIV-unknown partners $(\mathrm{N}=91)$ & $10(90.0)$ & $67(83.8)$ & 0.54 & $1.94(0.23,16.5)$ & $1.74(0.20,18.2)^{\mathrm{e}}$ \\
\hline
\end{tabular}

${ }^{a}$ Includes insertive or receptive anal intercourse at the most recent sexual encounter.

${ }^{\mathrm{b}}$ Adjusted for age, alcohol and drug use at last sexual encounter, and years since HIV diagnosis.

${ }^{\mathrm{c}}$ Adjusted for partner type and alcohol and drug use at last sexual encounter.

${ }^{d}$ Not calculated due to small number of subjects engaging in unprotected sex.

${ }^{\mathrm{e}}$ Adjusted for age and alcohol and drug use at last sexual encounter. 
protected sex with serodiscordant and serostatusunknown partners.

The rate of HIV disclosure in our cohort was low (26\%). Similar to studies in other countries [4], men whose most recent sexual partner was either a nonprimary or serodiscordant partner had the lowest rates of disclosure. We also found that younger men were less likely to disclose. Previous studies have found positive associations between HIV serostatus disclosure with other factors such as sexual orientation and HIV disease stage $[4,5]$; we did not find such associations in this study.

Since we did not explore the reasons for non-disclosure, it remains unclear why so few men in our study population chose to disclose. There could be multiple barriers to disclosing one's HIV serostatus to sexual partners, such as fear of rejection, lack of trust or intimacy with sexual partner, fear of stigmatization or discrimination, lack of self-efficacy for disclosure, or lack of feelings of personal responsibility to disclose, especially if protected sex is planned.

A major finding of this study was the general lack of an association between HIV serostatus disclosure and protected sex. The most commonly reported behavioral pattern in our population was to withhold disclosure while practicing protected sex. This "uniformed protection" may represent a compromise between a fear of negative consequences of disclosure (e.g., refusal to have sex, stigmatization, discrimination, loss of privacy) and a desire to not put one's sexual partner at risk [7], thereby highlighting the challenges associated with serostatus disclosure.

There were two interesting findings among men in seroconcordant relationships. As a group, men with a seroconcordant partner were less likely to have protected sex than men with a serodiscordant or serostatusunknown partner. But when they did report protected sex, it was associated with disclosure. This suggests that men in this group were less aware of or less concerned about risks such as HIV superinfection and transmission of drug resistant virus and thereby did not prioritize using condoms or mutually disclosing their HIV-positive serostatus.

This study has several limitations. First, the study population of clients who sought HIV testing at our center may not be representative of the general MSM population in Bangkok. Second, we only analyzed sexual behavior and disclosure practices with the most recent sexual partner, instead of examining these practices across multiple partners and sexual encounters. However, our strategy may have increased the reliability of subject responses and decreased recall bias [7]. Finally, we were limited by missing data, which accounted for a percentage of non-responders for most variables; however, the response rate to all of these variables was $88 \%$ or greater.

\section{Conclusions}

In conclusion, rates of HIV disclosure to sexual partners by HIV-positive MSM in Bangkok are low. Despite low rates of HIV serostatus disclosure, most HIV-positive MSM reported protected sex with their partners at risk for infection. Future studies should focus on understanding the barriers to discussion of serostatus amongst MSM and the factors that continue to drive unprotected sex in this population.

\section{Methods}

This was a cross-sectional questionnaire survey among 200 HIV-positive MSM receiving services from the Thai Red Cross Anonymous Clinic, an HIV voluntary counseling and testing center in Bangkok, Thailand. Inclusion criteria included male gender at birth, $\geq 18$ years of age, HIV-positive serostatus confirmed on prior testing, reporting either insertive and/or receptive anal sex with a male in the past 3 months, able to speak and read Thai, and having basic computer skills. The study protocol was approved by the Chulalongkorn University and Columbia University Institutional Review Boards. Eligible men were identified and recruited by the study nurse during their follow-up clinic appointments or initial walk-in visits at the Thai Red Cross Anonymous Clinic. Men were asked to complete a 20-minute behavioral questionnaire in Thai using Audio ComputerAssisted Self-Interviewing. Most recent CD4 T cell count (drawn at that visit or at the most recent visit prior to enrollment) was extracted from the clinic's laboratory database. Data collection occurred between March and June of 2010.

HIV serostatus disclosure to the most recent sexual partner prior to or at the time of sexual encounter was assessed by asking the question, "Did your most recent sexual partner know you are infected with HIV before you had sex?" (to assess partner awareness), followed by the question, "Did you discuss your HIV status with this person before you had sex?" An answer of "Yes, I told him/her I was HIV positive" was classified as disclosure; any other response was considered non-disclosure. Protected sex was defined as insertive or receptive anal intercourse with a condom at the most recent sexual encounter. Chi-square and Fisher exact tests were used in analyses to evaluate the association between protected sex and disclosure (with protected sex as the dependent variable) and the factors associated with disclosure (with disclosure as the dependent variable). Binary logistic regression models were developed, including covariates with $\mathrm{p}<0.15$ in the univariate analyses; adjusted odds ratios (OR) with 95\% confidence intervals (CI) were 
calculated. Statistical analysis was conducted with SPSS Version X (SPSS Inc, Chicago, IL, USA).

\section{Abbreviations}

ART: Antiretroviral therapy; MSM: Men who have sex with men.

\section{Competing interests}

The authors declare that they have no competing interests.

\section{Authors' contributions}

NEJ, Nittaya P, HVT, MES, PP and JA designed the study. NEJ, Nittaya P and JA drafted the protocol and the manuscript. NEJ, NC, NT, WS, CP, Nutthasun P carried out the study. NEJ performed the statistical analysis. All authors read, gave input and approved the final manuscript.

\section{Acknowledgements}

The authors would like to thank the participants and the staff at the SEARCH office and the Thai Red Cross Anonymous Clinic. The project was supported by the Doris Duke Clinical Research Fellowship Program and Infectious Diseases Society of America Medical Scholars Program through scholarships awarded to Nneka Edwards-Jackson.

\section{Author details}

${ }^{1}$ College of Physicians \& Surgeons, Columbia University, New York, USA. ${ }^{2}$ SEARCH, 104 Rajdumri Road, Pathumwan, Bangkok 10330, Thailand. ${ }^{3}$ The Thai Red Cross AIDS Research Center, 104 Rajdumri Road, Pathumwan, Bangkok 10330, Thailand. ${ }^{4}$ Laboratory of Infectious Disease Prevention, Lindsley F. Kimball Research Institute, New York Blood Center, New York, USA. ${ }^{5}$ Faculty of Medicine, Chulalongkorn University, Bangkok, Thailand.

Received: 10 October 2012 Accepted: 8 December 2012

Published: 23 December 2012

\section{References}

1. van Griensven F, Varangrat A, Wimonsate W, Tanpradech S, Kladsawad K, Chemnasiri T, Suksripanich O, Phanuphak P, Mock P, Kanggarnrua K, McNicholl J, Plipat T: Trends in HIV prevalence, estimated HIV incidence, and risk behavior among Men Who have Sex with Men in Bangkok, Thailand, 2003-2007. J Acquir Immune Defic Syndr 2009,

2. Centers for Disease Control and Prevention (CDC): HIV prevalence among populations of men who have sex with men-Thailand, 2003 and 2005. MMWR Morb Mortal Wkly Rep 2006, 55:844-848.

3. Crepaz N, Marks G: Serostatus disclosure, sexual communication and safer sex in HIV-positive men. AIDS Care 2003, 15:379-387.

4. Klitzman R, Exner T, Correale J, Kirshenbaum SB, Remien R, Ehrhardt AA, Lightfoot M, Catz SL, Weinhardt LS, Johnson MO, Morin SF, Rotheram-Borus MJ, Kelly JA, Charlebois E: It's not just what you say: relationships of HIV dislosure and risk reduction among MSM in the post-HAART era. AIDS Care 2007, 19:749-756.

5. Simon Rosser BR, Horvath KJ, Hatfield LA, Peterson JL, Jacoby S, Stately A: Predictors of HIV disclosure to secondary partners and sexual risk behavior among a high-risk sample of HIV-positive MSM: results from six epicenters in the US. AIDS Care 2008, 20:925-930.

6. Kalichman SC, Nachimson D: Self-efficacy and disclosure of HIV-positive serostatus to sex partners. Health Psychol 1999, 18:281-287.

7. Marks G, Crepaz N: HIV-positive men's sexual practices in the context of self-disclosure of HIV status. J Acquir Immune Defic Syndr 2001, 27:79-85.

8. Kinsey AC, Pomeroy WR, Martin CE: Sexual behavior in the human male. 1948. Am J Public Health 2003, 93:894-898.

9. Sittitrai W, Brown T, Sakondhavat C: Levels of HIV risk behaviour and AIDS knowledge in Thai men having sex with men. AIDS Care 1993, 5:261-271.

doi:10.1186/1742-6405-9-38

Cite this article as: Edwards-Jackson et al:: HIV serostatus disclosure is not associated with safer sexual behavior among HIV-positive men who have sex with men (MSM) and their partners at risk for infection in Bangkok, Thailand. AIDS Research and Therapy 2012 9:38. 